\title{
Rizo tomia e discectomia endoscópica no tratamento da dor lombar crônica e radiculopatia aguda na síndrome da pessoa rígida- relato de caso
}

\author{
Tatiane Bacchi Gehlen Lenzi de Araújo ${ }^{1}$ \\ Prof. Me. Jonas Lenzi de Araújo ${ }^{2}$ \\ Dra. Marcia Morete ${ }^{3}$ \\ Dr. Fabiano de Abreu Agrela Rodrigues ${ }^{4}$ \\ Bruno José de Pinho Miranda ${ }^{5}$
}

\section{RESUMO}

Introdução: A síndrome da pessoa rígida (SPR) é um distúrbio neurológico raro, descrito pela primeira vez em 1956 por Moersch e Woltman. O quadro clínico apresenta início insidioso de achados clássicos de espasmos dolorosos episódicos, hiperreflexia, rigidez muscular axial, progredindo lentamente para os músculos proximais dos membros. A hiperlordose dolorosa devido à rigidez da coluna lombar é uma característica diagnóstica

1-Médica, pós-gadruada em dor pelo Hospital Albert Einstein, dermatologia na Faculdade de Ciências Medicas de Minas Gerais, pós-graduanda em Medicina Regenerativa pela Unimax/Orthoregen; Membro da Sociedade Brasileira de Estudo da Dor (SBED)

2-Orthopedic Spine Surgeon, Fellow of Interventional Pain Practice (World Institute of Pain). Neuroscientist, Lean 6 Sigma Black Belt. Enrolled in Master's Program in Manufacturing Engineering. Founder/coordinator of Neuroscience and Applied Technology Committee (Brazilian Society for the Study of Pain - an International Association for the Study of Pain chapter).

3-Enfermeira, Doutora pela Faculdade de Medicina da USP, Especialista em Dor e Cuidados Paliativos, Certificada em "Muldisciplinary Pain Management" pela University of Sidney - Australia, Certificada em "Evaluating and Treatment Pain" pela Harvard Medical School - Boston; Membro da Associação Internacional para Estudo da Dor - IASP; Membro da Sociedade Brasileira para Estudo da Dor - SBED; Membro da American Society Pain Management Nursing

4-PhD, neurocientista, mestre psicanalista, biólogo, historiador, antropólogo, com formações também em neuropsicologia, psicologia, neurolinguística, neuroplasticidade, inteligência artificial, neurociência aplicada à aprendizagem, filosofia, jornalismo e formação profissional em nutrição clínica - Diretor do Centro de Pesquisas e Análises Heráclito; Chefe do Departamento de Ciências e Tecnologia da Logos University International, UniLogos; Membro da Federação Européia de Neurociências. Universidades em destaque: Logos University International, UniLogos, Nova de Lisboa, Faveni, edX Harvard, Universidad de Madrid.

5-Médico com especialização em Radiologia pela UFF, Pós-graduação em tratamento da Dor pelo Hospital Sírio-Libanês, Especialização em Medicina Intervencionista da Dor pela Clínica Singular, Pós-graduando em medicina esportiva pela Faculdade Paulista de Ciências da Saúde, Pós-graduando em Medicina Regenerativa pela Unimax/Orthoregen, Fellow of International Pain Practice (FIPP), Membro do Colégio Brasileiro de Radiologia (CBR), Sociedade Brasileira para Estudos da Dor (SBED), Sociedade Brasileira dos Médicos Intervencionistas em Dor (SOBRAMID), Sociedade Brasileira de Regeneração Tecidual (SBRET), Sociedade Brasileira de Terapias por Ondas de Choque (SMBTOC) 
do SPS. RELATO DO CASO: Homem, 69 anos, com SPS há 40 anos, encaminhado pelo neurologista ao cirurgião de coluna por falha no manejo conservador da lombalgia crônica, dor radicular progressivo na perna e alteração da marcha. O exame físico é difícil de realizar na SPR porque desencadeia espasmos. A ressonância magnética apresentou degeneração nas articulações zigapofisárias, nos discos intervertebrais e muscular, incluindo hérnia discal L4L5 com migração cranial. Uma vez que não havia outras maneiras eficazes de gerenciar sua dor axial e radicular, foi decidido realizar discectomia endoscópica transforaminal percutânea para descompressão de L4L5, rizotomia de radiofrequência ablativa do ramo medial do gânglio dorsal de L3 a S1 para aliviar a dor facetaria e rizotomia de radiofrequência pulsada do gânglio sensitivo dorsal L2 para modulação da dor lombar crônica. Após um ano de pós-operatório, o paciente apresentou melhora na EVA e no SF-36. CONCLUSÃO: A dor crônica relatada pelo paciente deve ser tratada nas dimensões biológica, psicológica e social. Compreender a multidimensionalidade dos fatores envolvidos com a dor permite planejar estratégias menos agressivas e altamente eficientes em pacientes incomuns e off label, como no caso do SPS.

Palavras-chave: síndrome da pessoa rígida; stiff-person; dor crônica; discectomia endoscópica; rizotomia ablativa; rizotomia pulsada; tratamento minimamente invasivo. 


\title{
Rhizotomy and endoscopic discectomy for chronic low back pain and acute radiculopathy in a stiff-person syndrome - case report
}

\begin{abstract}
Introduction: Stiff Person Syndrome (SPS) is a rare neurological disorder, first described in 1956 by Moersch and Woltman. The clinical picture has an insidious onset of classic findings of episodic painful spasms, hyperreflexia, axial muscle stiffness, progressing slowly to the proximal limb muscles. Painful hyperlordosis due to lumbar spine stiffness is a diagnostic characteristic of SPS. CASE REPORT: Male, 69 years old, with SPS for 40 years, referred by the neurologist to the spine surgeon because of failure to conservative management of chronic low back pain, progressively acute leg and gait impairment. Physical examination is difficult to perform in SPS because it triggers spasms. MRI presented zygoapophyseal joint, intervertebral discs and muscle degeneration, including a L4L5 cranial migrated herniated disc. Once there were no other effective ways to manage his axial and radicular pain, it was decided to perform percutaneous transforaminal endoscopic discectomy for L4L5 decompression, L3 to S1 dorsal ganglion root medial branch ablative radiofrequency rhizotomy to relieve facet pain and L2 dorsal sensory ganglion pulsed radiofrequency rhizotomy for modulation of chronic low back pain. One year postoperative follow up, the patient has showed improvement in the VAS and SF-36. CONCLUSION: Chronic pain reported by a patient must be addressed in the biological, psychological and social dimensions. Understanding the multidimensionality of the factors involved with pain allows planning less aggressive and highly efficient strategies in uncommon and off label patients, as in the case of SPS.
\end{abstract}

Keywords: rigid person syndrome; stiff-person; chronic pain; endoscopic discectomy; ablative rhizotomy; pulsed rhizotomy; minimally invasive treatment.

Artículo recibido: 02 enero 2022 Aceptado para publicación: 28 enero 2022 Correspondencia: deabreu.fabiano@gmail.com Conflictos de Interés: Ninguna que declarar 


\section{INTRODUÇÃO}

A síndrome da Pessoa Rígida, também conhecida como síndrome do homem rígido (StiffPerson Syndrome - SPS) ou síndrome de Moersch-Woltmann, é um distúrbio neurológico raro. Foi descrita pela primeira vez em 1956, quando Moersch e Woltman observaram a presença de rigidez dos músculos das costas, abdominais e da coxa em 14 pacientes[1]. Eles conduziram um estudo por um período de 32 anos para descrever suas descobertas de espasmos progressivos, flutuantes, rígidos e dolorosos que levavam a uma aparência de homem de madeira.

Costuma se apresentar de maneira esporádica, embora já tenham sido descritos casos familiares[2]. Afeta duas vezes mais o gênero feminino do que o masculino. Seus sintomas geralmente se manifestam entre a terceira e sétima décadas de vida, apesar de terem sidos relatados casos neonatais[2][3][4][5].

O quadro clínico tem início insidioso dos achados clássicos de espasmos dolorosos episódicos, hiperreflexia e rigidez muscular axial, progredindo lentamente para os músculos proximais dos membros. Também progride para outros músculos do corpo, como os músculos do tórax, causando dificuldades respiratórias. A hiperlordose dolorosa devido à rigidez da coluna lombar é uma característica diagnóstica da SPS[4].

Os espasmos são frequentemente desencadeados por sensações e emoções como medo, estímulo tátil ou auditivo inesperado[3]. Sintomas autonômicos como taquicardia, hiperidrose, alterações da pressão arterial, constipação e retenção urinária acompanham os espasmos[6].

As características funcionais do portador da SPS são a marcha lenta e desequilibrada, assim como incoordenação dos movimentos[7]. A perda insidiosa da flexibilidade do tronco e, posteriormente, da musculatura dos membros condiciona aos doentes um déficit funcional significativo, levando frequentemente a dependência de terceiros[2].

A fisiopatologia exata da SPS ainda permanece incerta[4]. Em aproximadamente $60 \%$ dos casos é comprovada a existência de distúrbios autoimunes através da presença de altos níveis de anticorpos séricos e no líquido cefalorraquidiano (LCR) contra a descarboxilase do ácido glutâmico (GAD), enzima limitadora da síntese do neurotransmissor inibitório ácido gama-amino-butírico (GABA)[5][3].

Classicamente, existem duas isoformas da GAD: GAD65 e GAD67. O primeiro está associado a SPS, diabetes mellitus, ataxia cerebelar e encefalite límbica[4]. A inibição da 
atividade do GAD65 pelo anti-GAD65 prejudica a síntese de GABA, levando a baixos níveis desse importante neurotransmissor inibitório no cérebro e no LCR[3].

A Síndrome da Pessoa Rígida é uma doença que ataca o sistema nervoso central, mas também tem manifestações neuromusculares. Ela causa rigidez e espasmos musculares progressivos. Os sintomas se manifestam da seguinte maneira: Contratura muscular, rigidez e espasmos progridem insidiosamente no tronco e abdome e, em menor grau, nos membros inferiores e superiores. A doença pode piorar progressivamente, levando a pessoa à uma incapacidade e rigidez de todo o corpo.

O diagnóstico é feito pelo reconhecimento dos sintomas e corroborado pelas sorologias, pela resposta ao diazepam e pelos resultados de estudos de EMG, que mostram a atividade elétrica da contração aparente normal. Atualmente, os estudos científicos revelam que a maior parte dos portadores desta enfermidade possuem anticorpos contra descarboxilase do ácido glutâmico (DAG), que é a enzima envolvida na produção do neurotransmissor inibidor de GABA (ácido gama-aminobutírico). No entanto, a doença pode ser desencadeada por três fatores, que são: Autoimune, Paraneoplásica e Idiopática (aquela que surge espontaneamente, sem que a sua origem seja conhecida).

O primeiro é comum em portadores de diabetes do tipo 1. A razão é porque autoanticorpos contra várias proteínas envolvidas na sinapses GABA afetam os neurônios que surgem no corno anterior da coluna. Sobre o segundo tipo, ele é encontrado em torno de 1 a $2 \%$ dos portadores da doença. Nesse caso, os anticorpos anti anfifisina estão presentes, enquanto os anti-DAG não. A síndrome da pessoa rígida é comumente associada a câncer de mama, mas também pode ocorrer em pacientes com câncer pulmonar, renal, tireoidiano ou colônico ou linfoma de Hodgkin.

Normalmente o tratamento é feito à base de Diazepam, pois alivia a rigidez muscular de forma mais consistente. Se este remédio não estiver dando os resultados esperados, os médicos podem receitar baclofeno, que é administrado por via oral ou intratecal. Há aqueles que usam corticoides, mas o problema é que eles apresentam muitos efeitos adversos a longo prazo. O procedimento à base de IGIV pode resultar em melhora duradoura por até um ano. Se os pacientes não respondem à IGIV pode-se sugerir rituximabe ou plasmaférese. 


\section{RELATO DE CASO}

Masculino, 69 anos, portador de SPS há 40 anos, encaminhado pelo neurologista ao consultório do ortopedista cirurgião de coluna com queixa de dor lombar baixa progressiva nos últimos 3 anos e irradiação progressiva a membros inferiores. Relatava dor tipo choque, contínua, irradiada para pernas, principalmente a esquerda. Sintomas pioravam ao decúbito dorsal e com a marcha.

O paciente afirmava sofrer de dor em toda sua coluna por muitos anos, a qual julgava ser decorrente da rigidez muscular secundário à SPS. Entretanto, era enfático em afirmar que a dor lombar que cursava com irradiação a membros inferiores era diferente da dor habitual das últimas quatro décadas. Embasava sua crença por meio da premissa de que a dor habitual aliviava após a as infusões de imunoglobulina, porém, de maneira oposta, a dor irradiada não sofria qualquer alteração com as infusões.

Apresentava dificuldade para deambular por mais de 10 metros, necessitando sentar-se ou agachar para alívio da dor, configurando a claudicação neurogênica. Relatou piora da coordenação motora e da sensibilidade abaixo de joelhos, predominando uma sensação de peso em suas pernas. Percebeu que movimentos de extensão lombar, situações de fadiga muscular, ingestão de alimentos ou bebidas muito gelados e mudança brusca de temperatura climática desencadeavam quadros de espasmos, que, por sua vez, atuavam como fatores de agravo dos sintomas.

À época de sua avaliação, já se submetera a amputações dos quintos dedos dos pés por deformidades em garra (claw toes) que evoluíram para osteomielite. Apresentava uma dieta com baixo teor de gordura e livre de lactose em consequência de uma colecistectomia prévia e dor abdominal pós-prandial, respectivamente. Trata ainda hipertensão arterial sistêmica com maleato de enalapril $10 \mathrm{mg}$ duas vezes ao dia e fibromialgia com pregabalina $75 \mathrm{mg}$ duas vezes ao dia. Foi tabagista por 15 anos, cerca de 20 cigarros ao dia. Atualmente está há 30 anos sem fumar.

Seu sono era fragmentado, tendo que acordar a cada 40 minutos por espasmos musculares ou apneia. A falta de um sono reparador piorava a sensação de fadiga, culminando com piores índices de qualidade de vida. Casado, era o único responsável por cuidar da esposa acamada, portadora de siringomielia e esclerose múltipla com tetraparesia. 
Como tratamento específico para SPS, usava baclofeno 20 a $40 \mathrm{mg}$ ao dia, infusões mensais de imunoglobulina G, ciclobenzaprina $15 \mathrm{mg}$, diazepam $10 \mathrm{mg}$ e a associação de diclofenaco com carisoprodol e cafeína quando tinha dor mais severa.

Na primeira consulta, indicou a intensidade da dor pela escala visual analógica (EVA) em 10. Sua qualidade de vida aferida pelo questionário SF36 foi de 20 para capacidade funcional, 100 para limitação por aspectos físicos, 0 para saúde mental, 55 para vitalidade, 68 para aspectos emocionais, 50 para aspectos sociais, 77 para dor e 35 para estado geral de saúde. (Ver tabela 1)

Ao exame físico apresentava hiperrreflexia generalizada (quatro membros e cutâneo abdominal), hipoestesia em membros inferiores e espasticidade muscular difusa com deformidades articulares, principalmente em tornozelos e pés. O simples toque durante a realização do exame físico desencadeava contraturas limitantes que eram facilmente visualizadas à ectoscopia. Cada avaliação física necessitava aquecer o consultório, as mãos e os equipamentos médicos. $\mathrm{O}$ aviso de cada passo do exame físico e a manipulação gentil e lenta eram formas de diminuir a espasticidade reflexa.

A ressonância nuclear magnética $(\mathrm{RNM})$ da coluna lombar demonstrava importante degeneração gordurosa da musculatura paravertebral, sinais de esclerose e hipertrofia facetária difusa, sobretudo em L3-L4, L4-L5 e L5-S1, derrame articular zigoapofisário L5-S1 com sinais de sinovite (figura 1), estenose foraminal L4-L5 bilateral às custas de hipertrofia facetária, de hipertrofia de ligamento amarelo, abaulamento do disco intervertebral L4-L5 e migração cranial de extrusão discal $(1,75$ x 1,56 x 0,6 cm) em recesso lateral direito, comprimindo a raiz emergente de L4 e descendente de L5 (figura 2). Também se identificava espondilolistese L4-L5 Meyerding grau I.

Figura 1: Imagem de RNM lombar ponderada em T2 axial demonstrando degeneração das facetas articulares L5-SI

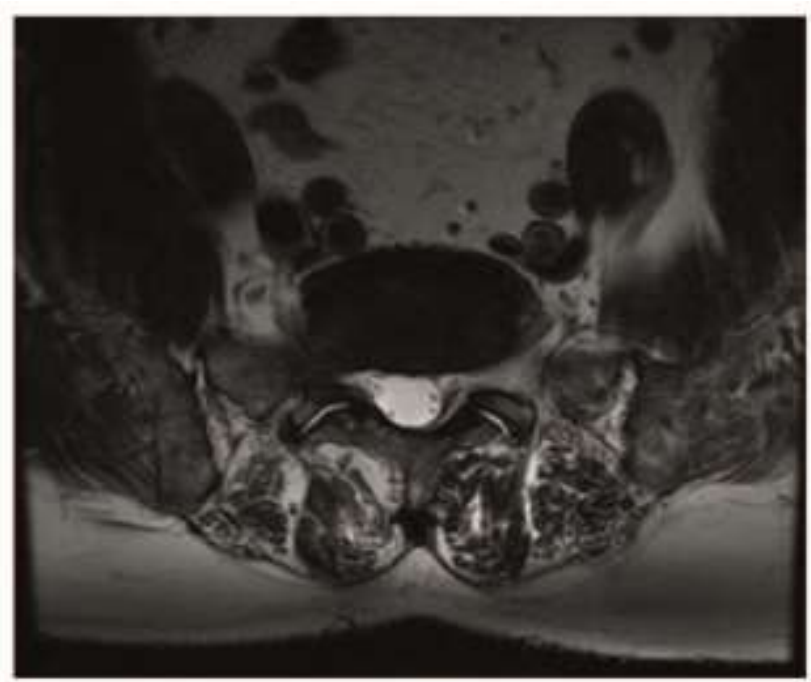


Figura 2: Imagem de RNM lombar ponderada em T2 axial (esquerda) e sagital (direita) demonstrando hérnia discal extrusa com migração cranial e compressão neural
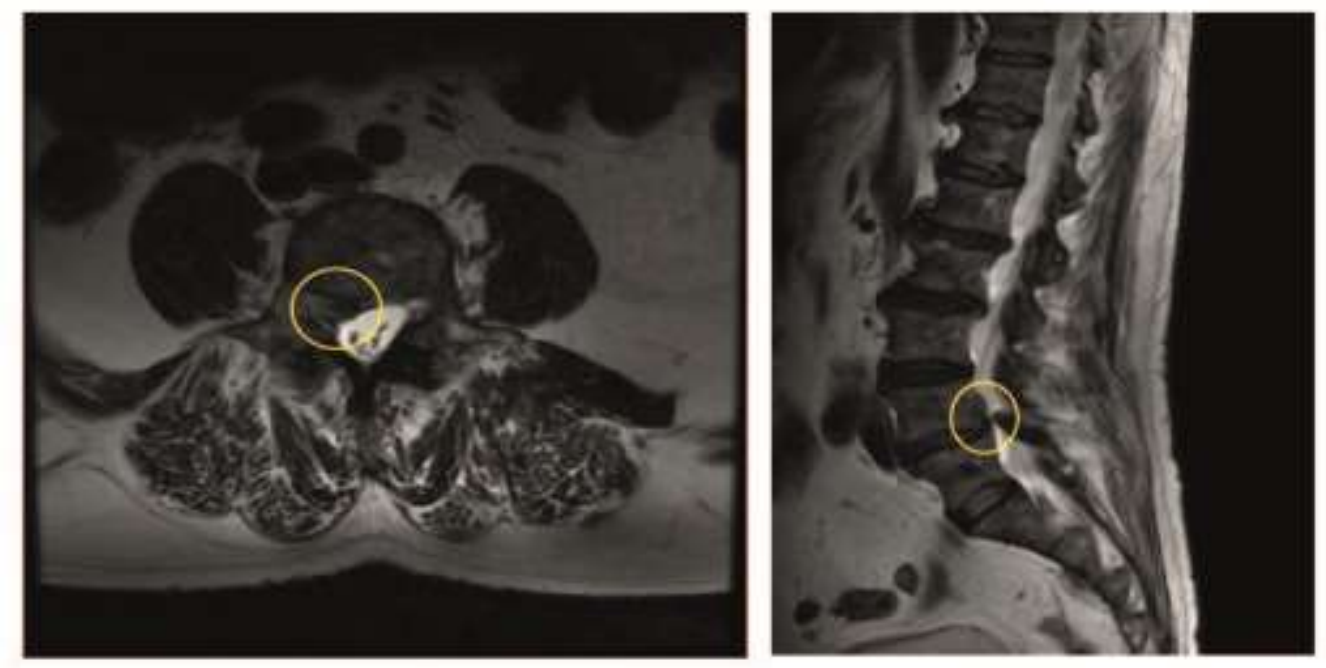

Como já fora encaminhado pelo neurologista, já se submetera a tratamento conservador com medicações e fisioterapia. Relatava agravamento dos sintomas com a reabilitação física porque tanto o deslocamento até a clínica quanto os estímulos exteroceptivos (tato, térmico, elétrico e vibratórios) recebidos pelos terapeutas desencadeavam espamos musculares.

Por não haver uma alternativa efetiva para tratamento conservador e o paciente sofrer de dor axial e radicular, foi decidido realizar discectomia endoscópica transforaminal percutânea para descompressão ocasionada pela hérnia de disco L4-L5, rizotomia por radiofrequência ablativa (A-RF) a $80^{\circ} \mathrm{C}$ (2 ciclos de 90 segundos) no ramo medial do gânglio dorsal de L3 a S1 para tratar a dor facetária e rizotomia por radiofrequência pulsada (P-RF) por 10 minutos a $42^{\circ} \mathrm{C} 5 \mathrm{~Hz}$ no gânglio sensitivo dorsal de L2 (dorsal root ganglia - L2-DRG) para modulação da dor lombar crônica.

Procedimento foi realizado com anestesia geral para garantir via aérea e pleno relaxamento muscular perioperatórios. A avaliação pela equipe anestésica foi de que o risco de disfunção ventilatória pela posição em decúbito ventral e contraturas tetânicas pela realização dos procedimentos sob sedação e anestesia local sobrepujavam o risco de uma anestesia geral. Não houve qualquer dificuldade técnica perioperatória, tendo duração aproximada de 90 minutos.

O paciente foi extubado logo após acordar na sala cirúrgica. Permaneceu curto período na sala de recuperação anestésica e foi transferido para unidade de tratamento intensivo 
(UTI). Sua estada na UTI foi de apenas 6 horas, sendo transferido para a enfermaria no fim do dia.

Com 12 horas pós-operatório (PO), o médico assistente visitou o paciente. Acompanhou o paciente para levantar-se e deambular. Deambulou por cerca de 200 metros, onde notou melhora significativa da dor, da sensibilidade, da força muscular e da coordenação motora em membros inferiores. No dia seguinte, com 24 horas PO, teve sua alta hospitalar com analgésicos simples e suas medicações de uso regular.

No retorno após 18 dias do procedimento cirúrgico, relatou melhora sustentada da força muscular em membros inferiores, marcha mais equilibrada e maior segurança durante a marcha. Ao exame físico apresentou ferida operatória cicatrizada, sem sinais de infecção. Era nítida a maior facilidade para realizar os movimentos básicos, como se levantar da poltrona, tirar sua roupa e deambular. Como não desejava fazer fisioterapia por causa do tempo despendido para deslocamento, tempo em que preferia se dedicar a sua esposa acamada, foi orientado a ao menos praticar atividades físicas domiciliares.

$\mathrm{Na}$ consulta após 4 meses da cirurgia, referiu melhora em torno de $60 \%$ da força em membros inferiores. Gabava-se por voltar a conseguir caminhar mais de 500 metros sem obstáculos. Mantinha o uso de pregabalina $75 \mathrm{mg}$ duas vezes ao dia, diazepam $10 \mathrm{mg}$ à noite, baclofeno $20 \mathrm{mg}$ à noite, infusões mensais de imunoglobulina $\mathrm{G}$, ciclobenzaprina e cloridrato de tramadol quando dor aumentava.

Seus escores da EVA e SF36 estão dispostos na tabela 1. Sua avaliação da dor pela escala visual analógica foi 1 e da qualidade de vida pelo questionário SF36 foi 45 para capacidade funcional, 100 para limitação por aspectos físicos, 0 para saúde mental, 85 para vitalidade, 84 para aspectos emocionais, 62 para aspectos sociais, 42 para dor e 45 para estado geral de saúde.

Tabela 1: Comparação das escalas de dor e qualidade de vida

\begin{tabular}{|l|c|c|}
\hline \multicolumn{1}{|c|}{ Escalas } & Pré-operatório & Pós-peratório \\
\hline EVA & 10 & 1 \\
\hline SF36- Capacidade funcional & 20 & 45 \\
\hline SF36- Limitação por aspectos físicos & 100 & 100 \\
\hline SF36- Saúde Mental & 0 & 0 \\
\hline SF36- Vitalidade & 55 & 85 \\
\hline
\end{tabular}




\begin{tabular}{|l|l|l|}
\hline SF36- Aspectos emocionais & 68 & 84 \\
\hline SF36- Aspectos sociais & 50 & 62 \\
\hline SF36- Dor & 77 & 42 \\
\hline SF36- Estado Geral de Saúde & 35 & 45 \\
\hline
\end{tabular}

Portava uma RNM realizada com 108 dias PO onde era possível identificar as mesmas alterações degenerativas em facetas articulares e musculares, persistindo o derrame facetário associado à sinovite. Porém, podia-se facilmente visualizar a descompressão radicular que comprovava a eficiência da discectomia endoscópica transforaminal percutânea em remover o componente discal extruso e migrado (figura 3).

Figura 3: Imagem de RNM lombar ponderada em T2 axial (esquerda) e sagital (direita) demonstrando a eficácia da discectomia endoscópica em remover o fragmento migrado. Abaixo, imagem com realce pelo gadolínio evidencia o sítio da discectomia L4-L5 e processo inflamatório perifacetário.
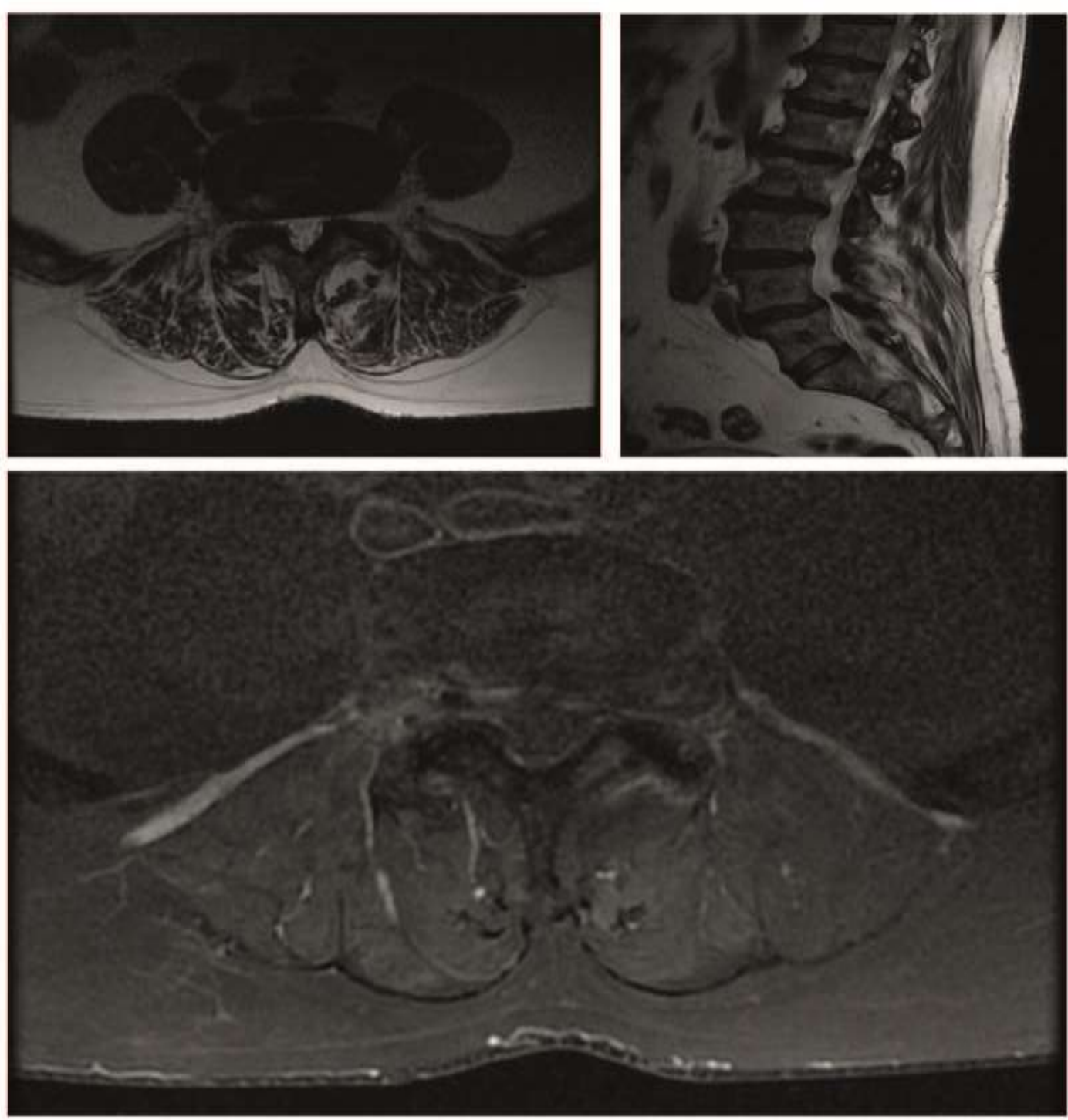

Ciencia Latina Revista Científica Multidisciplinar, Ciudad de México, México. ISN 2707-2207 / ISSN 2707-2215 (en línea), enero- febrero, 2022, Volumen 6, Número 1. 
Com 1 ano de seguimento paciente apresentava manutenção dos bons resultados obtidos desde o primeiro dia pós-operatório. Subjetivamente, ele julgava que a intensidade do alívio se sustentara quando comparado com 4 meses de seguimento, conforme contato por meios eletrônicos. Infelizmente não foi possível obter quantitativamente através das escalas EVA e SF-36 pois, como estava bem e tinha dificuldade para locomoção, não agendou mais consultas presenciais.

\section{DISCUSSÃO}

O tratamento da dor é um verdadeiro desafio a qualquer profissional. É bem comum um paciente chegar a um dado especialista com um diagnóstico estabelecido e clamar por resolução imediata. Vir com diagnóstico pré-estabelecido pode representar frequentemente um trabalho mais árduo a se executar.

É primordial que se entenda exatamente como a dor se comporta para posteriormente estabelecer um plano lógico de tratamento. Rotineiramente o plano idealizado é questionado pelos pacientes que já construíram crenças a partir de um doctor shopping que fornece informações diversas. Nesse ínterim, o paciente costuma acreditar na conduta que se enquadra melhor às suas expectativas, o que pode não ser a versão mais correta. Aí reside boa parte da dificuldade em tratar dor crônica. Porém cada paciente tem uma história única plena de experiências, assim como objetivos de vida claros.

Ao assumir um estereótipo caricato de um paciente com fibromialgia com dor generalizada, muitos casos potencialmente tratáveis podem ser condenados ao sofrimento permanente.

Claramente a SPS é uma doença crônica sem uma cura possível. O desequilíbrio muscular consequente pode ser a causa da dor crônica. É o protótipo da dor lombar mecânica ou dor lombar não específica altamente potencializada pela perda das vias inibitórias GABAérgicas. Pensando dessa maneira, a reabilitação física é imprescindível.

O paciente descrito apresenta um dado fundamental inerente a sua doença base: ele não tolera estímulos sensitivos por não apresentar o contrabalanço da atividade normal das vias inibitórias dependentes do GABA. Cada potencial de ação pode culminar com um verdadeiro curto-circuito em seu sistema nervoso central.

Considerando a quantidade de alterações degenerativas em sua coluna lombar, não restaria outra opção além do tratamento cirúrgico. Na ausência da SPS, possivelmente seria indicada um procedimento de descompressão e artrodese. Esse procedimento gera 
uma grande lesão tecidual e necessita de uma reabilitação mais intensa, o que não era tolerado pelo paciente com SPS.

O plano estabelecido foi identificar as principais possíveis causas para os diferentes tipos de dor presentes. Portanto o médico assistente assumiu a radiculopatia compressiva pela hérnia discal como principal causador da dor radicular com déficit neurológico e artrose zigoapofisária como principal causa de dor lombar axial. Não foi considerada a hipótese de realizar bloqueios anestésicos prévios como meio de identificar dor facetaria, sacroilíaca ou discogênica. A ideia era ser resolutivo em apenas um tempo para evitar levar o paciente múltiplas vezes ao centro cirúrgico.

Normalmente, quando um paciente com alto risco cirúrgico necessita de um procedimento, é desejado que ele seja minimamente invasivo, inclusive sob aspecto da anestesia. Baseado nesse conceito, seria prudente realizar os procedimentos propostos sob sedação e anestesia local, contudo há um risco muito grande de espasmos musculares simultâneos que poderiam cursar com hipoventilação em um paciente em decúbito ventral. Os estímulos térmicos pela radiofrequência, a manipulação extensa do dorso do paciente e o estímulo tátil vigoroso e contínuo pelo contato dos coxins poderiam funcionar como gatilhos para os espasmos tetânicos. Por esses motivos o anestesista optou por garantir a via aérea com intubação orotraqueal e o bloqueio muscular com curare.

Não há muito o que ser discutido a respeito da indicação da discectomia. Contudo, a técnica pelo qual ela pode ser realizada ainda é motivo de debate. A discectomia aberta costumava ser o padrão-ouro, porém ela requer maiores dissecção muscular, ressecção óssea (lâmina e faceta), ressecção de ligamento amarelo e retração da raiz do nervo espinhal. Esses passos aumentam o risco de complicações e a possibilidade de instabilidade vertebral[8][9].

Comparada à discectomia aberta, a cirurgia minimamente invasiva através da endoscopia, tanto transforaminal quanto interlaminar, preserva boa parte do acesso cirúrgico. Portanto, a recuperação seria mais rápida e menos sofrida. As cânulas utilizadas chegam ao sítio desejado através da divulsão de partes moles, sem necessidade de desinserir a musculatura. Ahn et al. identificaram escores da EVA muito mais baixos após a discectomia endoscópica, comparado a discectomia aberta. Os autores ainda notaram que a escala de incapacidade (Oswesrty Disability Index) no grupo que foi submetido a discectomia aberta tendeu a piorar após 6 meses pós-operatório[8]. Para o paciente 
relatado, a discectomia endoscópica percutânea transforaminal pareceu ser a melhor indicação pelos motivos discutidos acima.

A rizotomia por radiofrequência foi planejada tanto de maneira ablativa como pulsada modulatória. O objetivo da A-RF era eliminar os estímulos nociceptivos aferentes oriundos das facetas articulares.

A P-RF realizada no L2-DRG aplica estimulação elétrica que não ultrapassa os $42^{\circ} \mathrm{C}$, portanto não gera lesão tecidual significativa (Sluijter et al., 1998). Alguns autores descreveram lesão ultraestruturais nos níveis microscópico e subcelular (Cosman et al., 2005; Erdine et al., 2009). Essas lesões estão localizadas seletivamente nas fibras nociceptivas mais finas (fibras $\mathrm{C}$ e fibras A-delta), enquanto raramente são identificadas nas fibras sensoriais mais espessas, nociceptivas e não-nociceptivas (Erdine et al., 2009)[10][11]. Esses fenômenos podem ser os meios pelo qual a P-RF está associada a diminuição da percepção da dor lombar crônica.

Tsou et al realizaram uma análise prospectiva de 3 anos que mostraram que o tratamento com P-RF no L2-DRG é seguro e eficaz para o tratamento da dor lombar crônica. Concluiram que a P-RF proporcionou alívio a médio prazo da dor lombar[12].

Um outro ponto importante a ser considerado, a P-RF de gânglio sensitivo dorsal (DRG) tem sido utilizada de maneira adjuvante no manejo de espasticidade do paciente com lesão raquimedular. Se considerarmos a espasticidade da lesão raquimedular como uma consequência da atividade dos neurônios motores alfa sem controle modulatório do neurônio motor superior, na SPS a falta da inibição das vias GABAérgicas poderia ter algum benefício com P-RF do DRG.

Chang et al. descreveram dois casos de espasticidade por lesão raquimedular que obtiveram controle eficaz da espasticidade fásica e tônica. Os autores sugerem que a PRF DRG parece ter aumentado o controle inibitório do reflexo espinhal e ter diminuído a entrada aferente excitatória[10].

Outros autores relataram que o campo eletromagnético gerado pela radiofrequência de baixa frequência aprimora as várias vias inibitórias descendentes (Sluijter e Racz, 2002) e tem efeito de depressão a longo prazo (Cosman et al 2005)[10]. A depressão de longo prazo é um fenômeno envolvido na neuroplasticidade. Assim, ao tornar uma sinapse menos responsiva, diminui a entrada de potenciais de ação excitatórios associados com a nocicepção e a espasticidade reflexa. 
O motivo de ter como alvo o L2-DRG é baseado na proposta de Nakamura et al. de que a principal via aferente de dor dos discos intervertebrais inferiores é através da raiz do nervo espinhal de L2, presumivelmente por meio de aferentes simpáticos do nervo sinuvertebral[12]. Este nervo está associado a dor lombar discogênica, correspondendo a 26-39\% dos pacientes com dor lombar crônica[13]. No caso de degeneração de um disco intervertebral lombar, a dor é induzida por fatores químicos, fatores mecânicos, sensibilizacão periférica, sensibilização central e memórias associadas a dor.

Do ponto de vista psicológico, embora o paciente fosse portador de uma síndrome rara e muito incapacitante, ele morava com sua esposa que apresentava limitações mais severas. O senso de responsabilidade pelos cuidados de sua esposa era aliado importante para auxiliar a adotar estratégias de enfrentamento e lhe garantir uma resiliência admirável. O paciente era fortemente motivado e tinha uma postura diametralmente oposta à catastrofização comum em pacientes com fibromialgia.

Por considerar que o paciente apresentava inúmeros fatores que cursam com dor, além da SPS, não fora considerado pelo médico assistente como portador de fibromialgia. Esse diagnóstico fora estabelecido anos antes da consulta com o cirurgião de coluna que conduziu esse caso relatado.

\section{CONCLUSÃO}

A dor crônica relatada por um paciente deve ser abordada nos âmbitos biológico, psicológico e social. Aprofundando mais, no âmbito biológico, deve ser estabelecida a relação causal e temporal dos mecanismos mecânicos, bioquímicos e funcionais dos achados da anamnese, exame físico e exames complementares. Idealmente ainda deve ser investigado em um nível mais profundo a cinemática da dor.

Compreender a multidimensionalidade dos fatores envolvidos com a dor permite planejar estratégias menos agressivas e com alta eficiência em pacientes pouco convencionais, como no caso da SPS.

Concluindo, como demonstrado em outros estudos por diversos autores, a descompressão medular e radicular através da discectomia endoscópica transforaminal foi eficiente em controlar a dor radicular e a disfunção neural pela compressão, sem representar uma agressão tecidual que poderia ser gatilho para espasmos musculares. A rizotomia A-RF bloqueou a entrada de estímulos nociceptivos da artrose facetaria que sustentavam uma via de retroalimentação: dor-rigidez-dor. Por sua vez, a rizotomia P-RF se demonstrou 
eficiente em modular a dor crônica, possivelmente por múltiplas causas, incluindo a dor discogênica. Não tem como ser afastada a possibilidade da P-RF ter atuadado como fator de controle adicional da espasticidade muscular.

\section{REFERÊNCIAS}

P. E. M. Carrilho and N. Discipline, "EFFICACY OF LEVETIRACETAM IN A CASE OF STIFF-PERSON SYNDROME - CASE REPORT 1 INTRODUCTION The term 'stiff-man 'was first introduced by Moersch and Woltman in 1956 ( 1 ) to describe the clinical features of "progressive fluctuating muscular rigidity 'a," vol. 18, no. 1, pp. 68-72, 2014.

A. Silva, M. Almeida, and S. Teixeira, "Síndrome De Stiff-Person E Diabetes Mellitus Caso Clínico," Rev Port End Diabetes e Met, vol. 2, pp. 55-62, 2010.

A. C. R. de Cerqueira, J. M. F. Bezerra, M. Rozenthal, and A. E. Nardi, "Stiff-Person syndrome and generalized anxiety disorder," Arq. Neuropsiquiatr., vol. 68, no. 4, pp. 659-661, 2010, doi: 10.1590/s0004-282x2010000400036.

A. B. Bhatti and Z. A. Gazali, "Recent Advances and Review on Treatment of Stiff Person Syndrome in Adults and Pediatric Patients," Cureus, vol. 7, no. 12, 2015, doi: $10.7759 /$ cureus.427.

M. Á. Serra Valdés, E. Milán Ginjaune, M. García Viera, J. C. Laurencio Espinosa, L. Carnesolta Suárez, and J. L. Valdés Fuster, "El Síndrome del Hombre rígido o Síndrome de la persona rígida. Presentación de un caso," Rev. Habanera Ciencias Medicas, vol. 13, no. 5, pp. 719-727, 2014.

O. Yagan, K. Özyilmaz, A. Özmaden, Ö. Sayin, and V. Hanci, “Anestesia em paciente com síndrome da pessoa rígida," Brazilian J. Anesthesiol., vol. 66, no. 5, pp. 543545, 2016, doi: 10.1016/j.bjan.2013.02.002.

H. M. Meinck, K. Ricker, P. J. Hülser, E. Schmid, J. Peiffer, and M. Solimena, "Stiff man syndrome: Clinical and laboratory findings in eight patients," J. Neurol., vol. 241, no. 3, pp. 157-166, 1994, doi: 10.1007/BF00868343.

W. Ruan, F. Feng, Z. Liu, J. Xie, L. Cai, and A. Ping, “Comparison of percutaneous endoscopic lumbar discectomy versus open lumbar microdiscectomy for lumbar disc herniation: A meta-analysis," Int. J. Surg., vol. 31, pp. 86-92, 2016, doi: 10.1016/j.ijsu.2016.05.061.

H. S. Kim, B. Paudel, J. S. Jang, K. Lee, S. H. Oh, and I. T. Jang, "Percutaneous 
endoscopic lumbar discectomy for all types of lumbar disc herniations (LDH) including severely difficult and extremely difficult LDH cases," Pain Physician, vol. 21, no. 4, pp. E401-E408, 2018, doi: 10.36076/ppj.2018.4.e401.

M. C. Chang and Y. W. Cho, "Effects of pulsed radiofrequency on spasticity in patients with spinal cord injury: A report of two cases," Neural Regen. Res., vol. 12, no. 6, pp. 977-980, 2017, doi: 10.4103/1673-5374.208593.

F. Imani, H. Gharaei, and M. Rezvani, "Pulsed Radiofrequency of Lumbar Dorsal Root Ganglion for Chronic Postamputation Phantom Pain," Anesthesiol. Pain Med., vol. 1, no. 3, pp. 194-197, 2012, doi: 10.5812/kowsar.22287523.3768.

H. K. Tsou et al., "Percutaneous pulsed radiofrequency applied to the L-2 dorsal root ganglion for treatment of chronic low-back pain: 3-Year experience - Clinical article," J. Neurosurg. Spine, vol. 12, no. 2, pp. 190-196, 2010, doi: 10.3171/2009.9.SPINE08946.

B. Shayota et al., "A comprehensive review of the sinuvertebral nerve with clinical applications," Anat. Cell Biol., vol. 52, no. 2, pp. 128-133, 2019, doi: 10.5115/acb.2019.52.2.128. 